\title{
Pest Detection and Extraction Using Image Processing Techniques
}

\author{
Johnny L. Miranda, Bobby D. Gerardo, and Bartolome T. Tanguilig III
}

\begin{abstract}
Detection of pests in the paddy fields is a major challenge in the field of agriculture, therefore effective measures should be developed to fight the infestation while minimizing the use of pesticides. The techniques of image analysis are extensively applied to agricultural science, and it provides maximum protection to crops, which can ultimately lead to better crop management and production. Monitoring of pests infestation relies on manpower, however automatic monitoring has been advancing in order to minimize human efforts and errors. This study extends the implementation of different image processing techniques to detect and extract insect pests by establishing an automated detection and extraction system for estimating pest densities in paddy fields. Experiment results shows that the proposed system provides a simple, efficient and fast solution in detecting pests in the rice fields.
\end{abstract}

Index Terms-Automated pests management, image analysis object detection, object extraction.

\section{INTRODUCTION}

Rice is the most important and a primary source of food in Asia especially in the Philippines. However, rice may lose its quantity and quality when rice is attacked by different insect pests. Therefore, it is a top priority to find effective methods to reduce the level of their infestation in the paddy fields. In agriculture, pest control has always been considered as the most challenging task for farmers [1]. Most of the farmers used the traditional pest management methods which is the regular spray program based on schedules rather than the presence of insect pests on the paddy fields. These chemicals kill useful insects which eradicate pests in crops.

Assessing the density of the rice pest population in paddy fields is very important for pest forecasting decisions. Sticky traps are widely used to trap the insect pests. The trapped insects are brought to the laboratory for counting and identify manually. Usually, crop technicians identify and segregate the insects manually according to their species and count the major pests separately. The resulting counts are used to estimate the pest density in the paddy fields. However, multiple site and frequent counting of rice pests is time consuming and tedious for a crop technician. This can lead to low count accuracy and delays in obtaining accurate counts

Manuscript received January 25, 2014; revised March 13, 2014.

Johnny Miranda is with the College of Resource Engineering and Agricultural Mechanization, Pampanga State Agricultural University, Magalang, Pampanga, Philippines (e-mail: john_miranda9@yahoo.com).

Bobby D. Gerardo is with the Institute of Information and Communications Technology, West Visayas State University, Lapaz, Iloilo City, Philippines (e-mail: bgerardo@wvsu.edu.ph).

Bartolome T. Tanguilig III is with the College of Information Technology Education, Technological Institute of the Philippines, Quezon City, Philippines (e-mail: bttanguilig_3@yahoo.com). that can lead to poor decisions on rice pest management.

Due to the rapid development of digital technology, there is an opportunity for image processing technology to be used in the field of agricultural research which could help the researcher to solve a complex problem. Image analysis provides a realistic opportunity for the automation of insect pest detection. This study extends the implementation of image processing techniques to estimate pest densities in rice fields by establishing an automated detection system. Through this system, crop technicians can easily count the pests from the collected specimens, and right pests' management can be applied to increase both the quantity and quality of rice production. Using the automated system, crop technicians can make the monitoring process easier. Rice infestation may be easily detected and monitored with the use of a camera.

\section{REVIEW OF RELATED LITERATURES AND STUDIES}

\section{A. Current Methods Used by the Crop Technicians in Sampling Insect Pests in the Paddy Fields}

Currently, the population of insect pests and the damage on rice are generally randomly distributed that later aggregate in clumps [2]. That is why the sampling method was used by the farmers to achieve this goal because they used this to collect, count or inspect a small part of the pest population. It actually determines the trends in population of organism. Sampling method has two fundamental reasons; the research and the pest management decision-making, which normally requires a considerable time, efforts and costs as well. There are no universal sampling methods but this is carried out by using a variety of techniques and devices depending on the objective of the work. One of this is the effective use of pest surveillance and monitoring system which may result to efficient timing of interventions and reduce cost of production.

In the study of Carino, Kenmore and Dyck [3] there are several sampling techniques and devices for pest management decision-making; the light trap, that involves varying size sample which is good for comparing seasonal and yearly catches of insects, but catches are subject to changes in insect behavior and do not catch none flying insects; the sweep net (catching insect using fishnet), is a fast method, very economical, and good for sampling arthropods staying in canopy of rice, but it has human error due to variability and poor catch of arthropods at the base of the plant; tapping the rice, this is a sampling method that utilize a collecting pan with soap solution or oil with water to collect arthropods at the base and stem of the rice. After tapping, arthropods are identified and counted immediately in the field; the visual counting and data recording can be done on 
field but also subject to human error and very costly in labor; sticky trap is also economical, it measures insect movement and colonization but does not catch non flying insect; yellow pan trap is also economical; it measures insect migration, easy sorting and counting of samples, but the attraction is due to color stimulus and does not catch none flying insects.

\section{B. Feature Extraction and Image Processing}

Image processing is the analysis and manipulation of graphical images from sources such as photographs and videos. There are three main steps in image processing; first, is the conversion of captured images into binary values that a computer can process; second, is the image enhancement and data compression; and the third is the output step that consists of the display or printing of the processed image. Image processing is used in such applications as satellite weather mapping, machine vision, and computer-based pattern recognition. The novel feature extraction presented by Phinyomark, Limsakul and Phukpattaranont [4] for Electromyography signal used white gaussian noise to represent interference. Two novel mean and median frequencies were presented for robust feature extraction. The paper by Alsmadin, Omar, Noa and Almarashdeh [5], presented the development of a system that can recognize isolated pattern of interest on image based on the combination of robust features extraction. The system was used as classifier for fish image recognition and able to classify and categorize fish into poison or non-poison family.

\section{Real Life Application of Image Processing in the Fields of Agricultural Research}

Several attempts have been made to build automatic insect identification systems based on image analysis. The study conducted by Samantha and Ghosh [6] is concentrated to eight major insect pests based on the records of tea gardens of North Bengal Districts of India. The authors apply correlation based feature selection for the feature extraction and reduction, and incremental back propagation neural network as the neural network algorithm used for classifications.

Do, Harp and Norris [7] designed a computerized pattern recognition system for non-specialist in recognizing arachids and other arthropods to make the specimen identification easier and accurate. The researchers promote early insect pest detection in greenhouse crops in order to reduce pesticide use. The target application of this system is the detection of pests on plant organs such as leaves. The goal of their work is to define an innovative decision support system for in situ early pest detection based on video analysis and scene interpretation from multi-camera data. In this research, they used apriori algorithm to detect white flies and aphids, and follow a generic approach to design a system that can easily adapt different categories of bioagressors.

Al-Saqer [8] developed a neural network-based identification system for pecan weevils. They used image descriptors as input in the neural network to recognize the pecan weevil. The authors collected different images of pecan weevil and other insects found in the paddy fields. After collecting the images they converted them into binary and resized to $114 \times 134$ pixels. To process the data, they used different image processing techniques such as Regional Properties and Zernike Moments.

The paper presented by Zhu and Zhang [9] proposed a novel method to identify insects. They used integrated region matching and dual tree complex wavelet transform for image matching. The collected images of lepidopteran insects are reduced into $248 \times 200$ pixels and filtered using mean shift algorithm. The processed image is segmented in region based on k-mean algorithm using color feature. They used a pixel-wise segmentation to classify pixels into region according to their color properties.

\section{MATERIALS AND METHODS}

\section{A. Image Acquisition}

The experiments on pest detection were carried out in the paddy fields. The authors setup a network of wireless cameras (protected against water projection and direct sun light) together with the sticky traps to capture the insect pests. The cameras used are CISCO Linksys Wireless-G Internet Home Monitoring Camera which can capture 10 frames per seconds at 8 megapixel resolution. The captured images were processed using a local machine equipped with Intel i3 processor and 4 GB RAM. The architectural design of the proposed system is shown on Fig. 1.

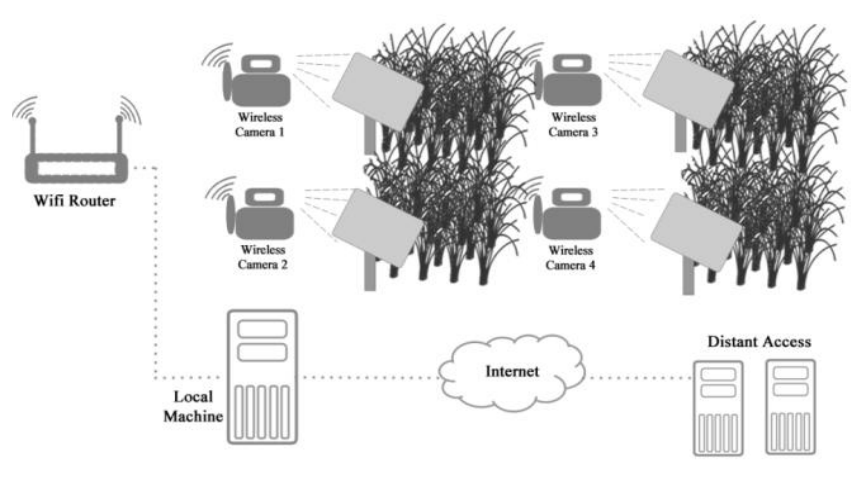

Fig. 1. Global architectural design.

\section{B. Image Pre-processing}

Image pre-processing creates an enhanced image that is more useful in processing the still image. In RGB color model, each color appears in its primary spectral components of red, green, and blue. The color of a pixel is made up of three components; red, green, and blue (RGB), described by their corresponding intensities. RGB color image require large space to store and consume much time to process. In image processing it needs to process the three different channels so it consumes large time. In this study, grayscale image is enough for the method so the authors convert the RGB image into grayscale image with the following formula:

$$
I(x, y)=0.2989 \times R+0.5870 \times G+0.1140 \times B
$$

\section{Detection of Pests in the Image}

The detection mechanism used to detect the insect pests in the image is simple and yet efficient. The authors compared the image pixel values of the successive captured images from the camera. Two images are used in detecting the difference. The first image served as the reference image that represents the reference pixel values for comparison purposes, while the second image served as the input image. The two images were compared to each other and the differences in pixel values were determined. If the inputted image pixel 
values are not equal to the reference image pixel values, the inputted image pixel will be saved as the output image pixel. If the pixel value of the input image is equal to the reference image then the background will be white (pixel value is 255). Fig. 2 shows the difference between reference and input image. After determining the difference of the reference and input image, the input image will now be used as the reference image and the output image will be the extract and served as the input on the next phase.

To determine the difference of the reference and input image, the following equation will be used:

$$
O_{R I}(x, y)= \begin{cases}255, & \text { if } R(x, y)=I(x, y) \\ I(x, y), & \text { if } R(x, y) \neq I(x, y)\end{cases}
$$

where $I(x, y)$ is the input image and $R(x, y)$ in the reference image in coordinate $(x, y)$.

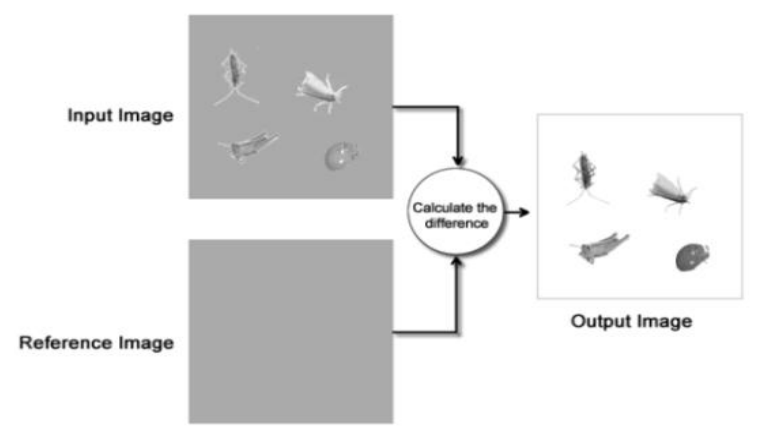

Fig. 2. Determining the difference of the reference and input image.

To demonstrate the difference between the reference $R(x, y)$ and input image $I(x, y)$, assume that we have two $4 \times 4$ grayscale images. The two images are represented in the following matrices:

$$
\begin{aligned}
& R(x, y)=\left[\begin{array}{llll}
230 & 230 & 230 & 230 \\
230 & 230 & 230 & 230 \\
230 & 230 & 230 & 230 \\
230 & 230 & 230 & 230
\end{array}\right] \\
& I(x, y)=\left[\begin{array}{cccc}
230 & 230 & 230 & 230 \\
230 & 30 & 22 & 230 \\
230 & 24 & 23 & 230 \\
230 & 230 & 230 & 230
\end{array}\right] \\
& O(x, y)=\left[\begin{array}{cccc}
255 & 255 & 255 & 255 \\
255 & 30 & 22 & 255 \\
255 & 24 & 23 & 255 \\
255 & 255 & 255 & 255
\end{array}\right]
\end{aligned}
$$

where the $O(x, y)$ is the output image in coordinate $(x, y)$.

\section{Filtering of the Image}

Filtering is a process of cleaning up the appearance of the image from noise caused by different lighting conditions. Digital image processing required filtering to yield a usable and attractive end result. There are different techniques available and the best options depend on the image and how it will be used. In this study the authors use median filter. Median filter looks at its nearby neighbor's pixel values to decide whether or not it is representative of its surrounding pixels and replaces with the median of those values. Fig. 3 illustrates an example calculation.

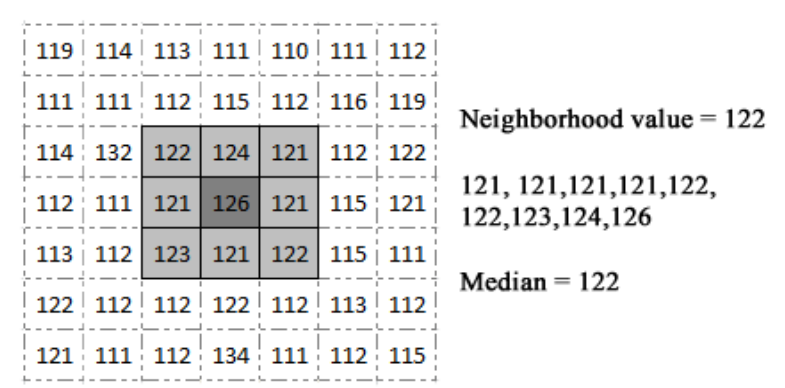

Fig. 3. Calculates the median value of a pixel neighborhood. The selected pixel value of 126 is replaced with median value of 122 because it is unrepresentative of the surrounding pixels.

\section{E. Extraction of the Detected Pests}

This phase is the extraction of the detected insect pest from the image. The output image which was obtained at the end of the previous phase was used in this phase. The image pixel values of the output image will be scanned both horizontally and vertically to determine the coordinates of each insect in the image. The width and height of the extracted image was determined by using its starting and ending coordinates. Once the start and end coordinates of the objects are determined, the width and height of the matrix as defined by those coordinates will take note and the matrix will be saved.

The method used in extracting has two steps. First, the value of the output image in pixels is scanned horizontally which starts at the first $x$-direction, then the pixel values are summed up to the corresponding columns. The coordinate value in $x$-direction is incremented by one and the total pixel value in the next column is calculated. The process is repeated until reaching the last value in the $x$-direction. As a result, the total pixel values of each column are calculated. In order to determine the $x$-coordinate where an object starts or ends within the image, each total value is compared to a certain threshold value. Second, it will vertically repeat the horizontal scanning method; therefore the total pixel value in each row is calculated. Then, to determine the $y$ coordinates, the thresholding will apply where objects start or end within the image. The mathematical description of the object extraction method is shown in the following equations.

Starting at coordinates $(x=0, y=0)$ until coordinate $\left(x=n_{x}\right.$, $y=n_{y}$ ), the total pixel value of the column and row are defined as:

$$
\begin{aligned}
& \operatorname{TotalY}_{x}=\sum_{y=0}^{n_{y}} O(x, y) \\
& \operatorname{TotalX}_{y}=\sum_{x=0}^{n_{x}} O(x, y)
\end{aligned}
$$

The comparison rules are defined as follows:

$$
\begin{array}{ll}
S_{x}=y & \text { if } \operatorname{TotalY}_{x} \leq \theta \\
E_{x}=y & \text { if } \operatorname{TotalY}_{x}>\theta \\
S_{y}=y & \text { if } \operatorname{TotalX}_{y} \leq \theta \\
E_{y}=y & \text { if TotalX } \\
\text { Tot } & >\theta
\end{array}
$$

where $\theta$ is the threshold, $S_{x}$ and $E_{x}$ are respectively the starting and ending $x$ coordinates of the object image and $S_{y}$ and $E_{y}$ are respectively the starting and ending y coordinates of the object image.

The size of the extracted object image is $\left(E_{x}-S_{x}\right) \cdot\left(E_{y}-S_{y}\right)$. The application of this algorithm continues until the row at the last y-coordinate pixel is reached. 


\section{EXPERIMENT RESULTS}

This section presents the experiment results after testing the proposed system. It explains first the experimental setup then shows the results on different test.

The proposed pest detection and extraction system was tested in the practice farm of Pampanga State Agricultural University, Magalang, Pampanga, Philippines. The area has 50x50 meters wide. The specimens were collected on the sticky traps that were captured by the 4 wireless cameras installed in the paddy field. The cameras were used to observe the sticky traps which capture a still image every 1 minute. These images were used both in the development and system testing.

The proposed pest detection system based on image processing techniques was tested in five consecutive days in the paddy field and was found efficient. The result is shown on Table I.

TABLE I: THE Detected IMAges of DifFERENT InSECT PeStS IN FiVE CONSECUTIVE DAYS

\begin{tabular}{lccccc}
\hline \hline & Camera & Camera & Camera & Camera & Total \\
& 1 & 2 & 3 & 4 & \\
\hline Day 1 & 15 & 12 & 13 & 10 & 50 \\
Day 2 & 12 & 10 & 12 & 10 & 44 \\
Day 3 & 10 & 13 & 12 & 11 & 46 \\
Day 4 & 14 & 15 & 10 & 13 & 52 \\
Day 5 & 12 & 11 & 10 & 5 & 38 \\
\hline \hline
\end{tabular}

\section{CONCLUSION}

In this paper, an automatic detection and extraction system was presented, different image processing techniques were used to detect and extract the pests in the captured image. The presented system is simple and yet efficient. The authors used background modeling to detect the presence of insect pests in the captured image, and a median filter was used to remove the noise produced by different lighting conditions. The mechanism used to extract the detected objects from the image is simple, the image was scanned both horizontally and vertically to determine each coordinates and save the object image.

The result presented in this paper is promising but several improvements on both materials and methods will be carried out to reach the requirements of fully automated pest detection, extraction and identification system. In the future, other image processing techniques may be used to enable the detection and extraction more efficient and accurate. Other future work may include the identification system of the extracted objects. This time a neural network mechanism will be used to identify and classify the detected pests.

\section{REFERENCES}

[1] Resource Manual on Integrated Production and Pest Management (IPPM) in Rice, World Education (INGO) Philippines, Inc., 2005, ch. 6, pp. 113-115.

[2] Sampling insect pests, their damage and beneficial organisms Philippine rice self-sufficiency plan (PRSSP). [Online]. Available: http://pinoyrkb.com/main/resources/publications-and-figures/rice-scie nce-for-decisionmakers/doc_download/517-sampling-insect-pests

[3] F. A. Carino, P. E. Kenmore, and V. A. Dyck, "A FARMCOP suction sampler for hoppers and predators in flooded rice fields," The International Rice Research Newsletter, vol. 4, ch. 5, pp. 21-22, 1979.
[4] A. Phinyomark, C. Limsakul, and P. Phukpattaranont, "A novel feature extraction for robust EMG pattern recognition," Journal of Computing, vol. 1, pp. 71-80, Dec. 2009.

[5] M. K. Alsmadin, K. B. Omar, S. A. Noah, and I. Almarashdeh, "Fish recognition based on robust features extraction from color texture measurements using back-propagation classifier," Journal of Theoretical And Applied Information Technology, 2010.

[6] R. K. Samanta and I. Ghosh, "Tea insect pests classification base on artificial neural networks," International Journal of Computer Engineering Science, vol. 2, June 2012.

[7] M. T. Do et al., "A test of a pattern recognition system for identification of spiders," Bulletin of Entomological Research, 1999.

[8] S. M. Al-Saqer, "A robust recognition system for pecan weevil using artificial neural networks," American Journal of App. Sci., 2012.

[9] L. Zhu and Z. Zhang, "Insect recognition based on integrated region matching and dual tree complex wavelet transform," Journal of Zhejiang University-SCIENCE C (Computers \& Electronics), 2011.

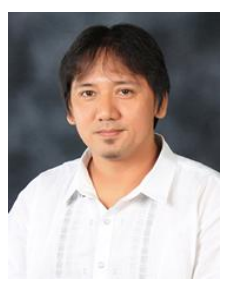

Johnny L. Miranda received BS in information technology and masters in information technology on 2002 and 2007. Currently, he is pursuing his doctora studies in information technology at Technological Institute of the Philippines, Quezon City. His research interest includes data mining, image processing, artificial intelligence and database.

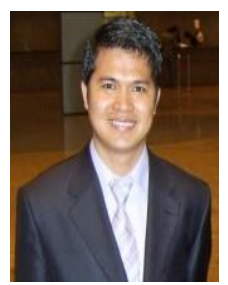

Bobby D. Gerardo is currently the vice president of Administration and Finance of West Visayas State University, Iloilo City, Philippines. His dissertation is "Discovering driving patterns using rule-based intelligent data mining agent (RiDAMA) in distributed insurance telematic systems." He has published 54 research papers in national and international journals and conferences. He is a referee of international conferences and journal publications such as IEEE Transactions on Pattern Analysis and Machine Intelligence and IEEE Transactions on Knowledge and Data Engineering. He is interested in the following research fields: distributed systems, telematics systems, CORBA, data mining, web services, ubiquitos computing and mobile communications.

Dr. Gerardo is a recipient of CHED Republica Award in Natural Science Category (ICT field) in 2010. His paper entitled "SMS-based automatic billing system of household power consumption based on active experts messaging" was awarded best paper on December 2011 in Jeju, Korea. Another best paper award for his paper was "Intelligent decision support using rule-based agent for distributed telematics systems," presented at the Asia Pacific International Conference on Information Science and Technology, on December 18, 2008. An excellent paper award was given for his paper "Principal component analysis mechanism for association rule mining," on Korean Society of Internet Information's (KSII) 2004 Autumn Conference on November 5, 2004. He was given a university researcher award by West Visayas State University in 2005.

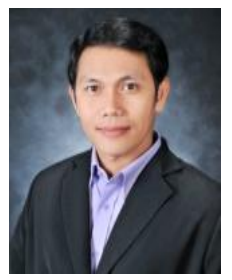

Bartolome T. Tanguilig III was born on February 24, 1970 in Baguio City, Philippines. He took his bachelor of science in computer engineering in Pamantasan ng Lungsod ng Maynila, Philippines in 1991. He finished his master degree in computer science from De la Salle University, Manila, Philippines in 1999. His doctor of philosophy in technology management was awarded by the Technological University of the Philippines, Manila in 2003.

$\mathrm{He}$ is currently the assistant vice president of Academic Affairs and concurrent dean of the College of Information Technology Education and Graduate Programs of the Technological Institute of the Philippines, Quezon City. His research entitled "J-master: an interactive game-based tool for teaching and learning basic java programming" was awarded the best research in the $10^{\text {th }}$ National Convention for IT Education held in Ilocos Norte, Philippines in 2012. He published a research entitled "Predicting faculty development trainings and performance using rule-based classification algorithm" in Asian Journal for Computer Science and Information Technology.

Dr. Tanguilig is a member of Commission on Higher Education Technical Panel for IT Education, Board Chairman of Junior Philippine IT Researchers, member of Computing Society of the Philippines and Philippine Society of IT Educators-NCR. 